\title{
Médiévales
}

Langues, Textes, Histoire

76 | printemps 2019

Du nouveau en archives

\section{De la « grande crise » à la « grande transition » : une nouvelle perspective?}

From the "great crisis" to the "great transition": A new perspective?

Jean-Philippe Genet

\section{OpenEdition}

1 Journals

Édition électronique

URL : https://journals.openedition.org/medievales/10046

DOI : 10.4000/medievales. 10046

ISSN : 1777-5892

Éditeur

Presses universitaires de Vincennes

Édition imprimée

Date de publication : 5 septembre 2019

Pagination : 153-166

ISBN : 978-2-37924-028-7

ISSN : 0751-2708

\section{Référence électronique}

Jean-Philippe Genet, « De la " grande crise » à la " grande transition » : une nouvelle perspective ? », Médiévales [En ligne], 76 | printemps 2019, mis en ligne le 01 janvier 2021, consulté le 24 avril 2022. URL : http://journals.openedition.org/medievales/10046; DOI : https://doi.org/10.4000/medievales 10046

Tous droits réservés 
Jean-Philippe Genet

\section{De la " grande crise " à la " grande transition " : une nouvelle perspective?}

Dans sa préface à la réédition de son Histoire des climats, Emmanuel Le Roy-Ladurie soulignait l'ampleur des progrès accomplis dans le domaine de l'histoire du climat ${ }^{1}$. Les méthodes et les instruments de mesure se sont transformés, les données paléo-climatiques, mieux réparties et plus précises, se sont multipliées. Il est surtout possible de les faire entrer en résonance avec d'autres domaines de la science, biologie, médecine, botanique ou zoologie, pour définir une approche pluridisciplinaire et aboutir à une histoire renouvelée des sociétés humaines globale à double titre, à l'échelle du monde et sur le temps long : les données sur la nature s'ajoutent ainsi à celles dont on disposait sur les cultures et les sociétés. Mais comment saisir cette globalité nouvelle ? Faut-il l'exprimer par le concept d'histoire environnementale, comme Richard Hoffmann, professeur émérite à l'université d'York (Canada) ${ }^{2}$ ? Bruce M. S. Campbell, professeur à Belfast, préfère insister sur la combinaison des histoires du climat et de la maladie comme clés de compréhension du changement des conditions naturelles auxquelles s'adaptent nécessairement les sociétés humaines ${ }^{3}$.

Leurs deux livres concernent l'un et l'autre l'Europe médiévale mais dépassent ce cadre spatial et chronologique, même si Campbell met l'accent sur l'Angleterre et la période $\mathrm{XIII}^{\mathrm{e}}-\mathrm{XV}^{\mathrm{e}}$ siècle : les changements dont il est ici question n'ont de frontière ni dans le temps ni dans l'espace. Mais, en dépit du rôle essentiel qu'ils attribuent l'un et l'autre au climat, les deux livres sont fort différents. Les deux historiens explicitent les théories sousjacentes à leur démonstration par un diagramme de Venn. Celui de Richard

1. E. Le Roy-LAdURIE, Histoire du climat depuis l'an mil, Paris, 2009 [1983].

2. R. C. Hoffmann, An Environmental History of Medieval Europe, Cambridge, 2014.

3. B. M. S. CAMPBELL, The Great Transition. Climate, Disease and Society in the LateMedieval World, Cambridge, 2016. 
Hoffmann (Figure 1) présente deux sphères, la nature et la culture, chacune ayant son régime de causalité, à l'intersection desquelles sont placés les humains et les structures biophysiques de leurs sociétés : l'interaction entre elles passe par deux circuits, l'un mettant en relation la culture symbolique et les écosystèmes naturels et/ou colonisés par l'intermédiaire de la culture matérielle, l'autre agissant par une dialectique circulaire (programmes d'action $\rightarrow$ travail $\rightarrow$ expérience $\rightarrow$ représentation $\rightarrow$ programmes, etc.). Le diagramme de Campbell (Figure 2), dérivé de celui sur les menaces microbiennes adopté par l'Institut de médecine des États-Unis en 2003 et 2008, est à la fois plus complexe et plus radical : il prend la forme d'un ensemble de six carrés, les deux carrés extrêmes, qui n'ont pas d'intersection, étant le climat (et non la nature) et la société (et non la culture) : climat et société n'interagissent que par l'intermédiaire des quatre autres carrés, les écosystèmes, la biologie, les microbes et les humains. Et à l'intersection de ces quatre carrés est l'espace crucial où humains et microbes vivent une relation réglée par les écosystèmes et la biologie, eux-mêmes dépendant des évolutions du climat et des sociétés. Le modèle est complexe, mais la multiplicité des interactions le met à l'abri du déterminisme.

\section{Une histoire environnementale}

Richard Hoffmann écrit une histoire environnementale du Moyen Âge qui est une sorte de manuel foisonnant pour le public étudiant, sans notes et avec une bibliographie restreinte, lacunes que l'abondance des cartes et des illustrations est censée compenser. Il propose d'abord un parcours chronologique allant de Rome ${ }^{4}$ à la fin de l'époque carolingienne, dans lequel les principaux caractères de l'espace agraire et du climat du premier millénaire sont présentés, deux excursus détaillés étant consacrés à la Frise et à Venise, deux cas d'adaptation à des situations où l'eau peut contrarier les activités humaines. À ce stade, un chapitre entier est consacré aux conceptions médiévales de la relation entre homme et nature, à partir des théories de Lynn White $\mathrm{Jr}^{5}$ et de David Herlihy ${ }^{6}$, opposant deux perceptions de la nature, salvatrice ou hostile. Mais passer en revue en une vingtaine de pages denses les idées des théologiens d'Augustin à Albert le Grand et

4. Présentation générale dans J. BRoOKE, Climate Change and the Course of Global History, New York, 2014. Pour Rome, voir K. HARPER, The Fate of Rome. Climate, Disease, and the End of an Empire, Princeton, 2017 (trad. fr. Comment l'Empire romain s'est effondré. Le climat, les maladies et la chute de Rome, Paris, 2019).

5. L. White Jr., «The Historical Roots of Our Ecological Crisis », Science, 155 (10 mai 1967), p. 1203-1207 [en ligne] URL : http://science.sciencemag.org/content/155/3767/1203.

6. D. Herlihy, «Attitudes toward the Environment in Medieval Society », dans L. J. BILSKY éd., Historical Ecology : Essays on Environment and Social Change, Port Washington, 1980, p. 100-116. 


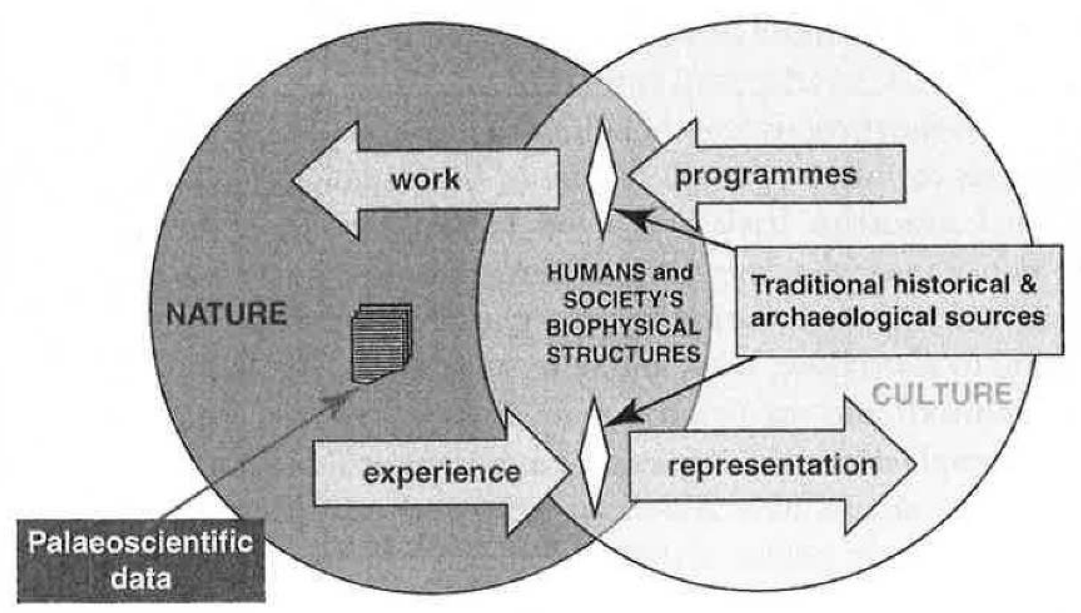

Fig. 1. Diagramme de Richard Hoffmann (le $5^{\mathrm{e}}$, présentant les sources de l'histoire environnementale)

Source : R. C. HofFMAnN, An Environmental History of Medieval Europe, Cambridge, 2014

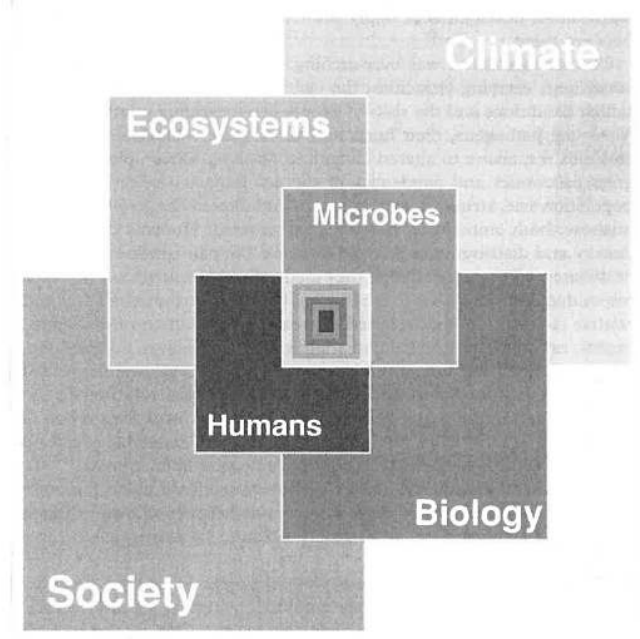

Fig. 2. Diagramme de Venn de Bruce Campbell

Source : B. M. S. CAMPBELL, The Great Transition. Climate, Disease and Society in the Late-Medieval World, Cambridge, 2016, p. 22. 
d'auteurs témoignant d'une expérience de la nature (Frédéric II, Gaston Phoebus, Piero de Crescenzi, etc.) est une gageure : l'auteur admet que, faute d'études systématiques des sources médicales, juridiques et didactiques, il n'est possible ni d'expliquer ni même de confirmer la réalité du gouffre qui sépare les conceptions médiévales de la nature de l'expérience qu'en ont les hommes.

Trois chapitres portent ensuite sur l'interaction entre la culture et les composantes organiques (l'origine des paysages et la formation des écosystèmes européens) et inorganiques (l'énergie - éolienne, hydraulique ou fossile - et les mines) de la nature et les activités qui en dépendent (transport, manufacture, vie urbaine). Là encore, la nouveauté de la perspective frappe plus que le contenu. Signalons aussi le recours à la notion de « paysage » chère à l'écologie historique et le choix original des exemples (ainsi les défrichements autour de Wroclaw, région des premières recherches de l'auteur). L'essor démographique et les grands défrichements sont omniprésents. Les «paysages » céréaliers méditerranéens, les terres irriguées et les terres humides de l'Europe du Nord (souvent négligées) font partie des « agroécosystèmes anthropogéniques médiévaux » qui entraînent des transformations imprévues de la biodiversité. Au total, l'impression dominante est celle d'un " processus de colonisation [...] qui transforme l'Europe en une mosaïque d'écosystèmes artificiels, pour la plupart conçus pour maximiser la production céréalière ». La « céréalisation »a permis de nourrir une population qui triple entre 800 et 1300 , mais ces systèmes répondaient d'abord aux exigences de la «culture » (opposée ici à « nature »: Hoffmann insiste sur ce point), en l'occurrence celle des seigneurs, les paysans opérant sous la contrainte socio-politique de leur pouvoir. On peut se demander s'ils n'avaient pas d'effets néfastes sur la nature : quant à l'usage des sols, les agroécosystèmes du Nord de l'Europe, tel l'openfield avec assolement et vaine pâture, paraissent stables, l'enrichissement des sols et la diversification des cultures les ayant améliorés, en dépit de crises dues aux accidents climatiques ou aux surexploitations (Hollande). La réponse est moins assurée pour l'Europe méditerranéenne dans l'état présent de la recherche. L'économie pastorale, la forêt et la chasse font l'objet de traitements spécifiques. Plus neuve est la partie sur les « écologies urbaines », sujet peu traité en tant que tel : les données sont lacunaires, pourtant l'auteur semble imputer à l'essor urbain la mise en péril des équilibres fondamentaux qui, si elle ne les détermine pas, est évidente dans les crises du XIV ${ }^{\mathrm{e}}$ siècle. Quantitativement, le rôle joué par les composantes inorganiques reste faible : on a affaire « à une société essentiellement agraire qui a colonisé la biosphère pour exploiter le 
flux de l'énergie solaire ${ }^{7}{ }^{\text {» }}$, et c'est sur cette base qu'il convient de juger sa capacité à s'adapter et à survivre.

Si les hommes du Moyen Âge restent «sujets aux forces puissantes de la sphère naturelle », ce qu'illustre leur attitude face à la maladie, aux tremblements de terre ou aux violences climatiques, ils ont conscience du respect dû aux exigences de la nature, et l'exploitation des ressources naturelles est très tôt régulée par les communautés paysannes et les seigneurs pour les défrichements et les assolements, et dès le XII ${ }^{\mathrm{e}}$ siècle, par les États naissants, d'abord pour les forêts puis pour les eaux, protection des rivières et des pêcheries notamment. Si l'on ne peut discerner de stratégie environnementale d'ensemble, les adaptations sont constantes, dans tous les domaines, économiques, sociaux (long développement sur le déclin des pratiques communautaires au profit de la propriété individuelle) ou politiques (contrôle des eaux et des forêts par les princes). Sur un strict plan écologique, le modèle médiéval n'a pas atteint ses limites et, en dépit de ses crises (en partie liées, on l'a vu, à la pression de l'essor urbain), il n'a pas été bloqué ; renvoyant dos à dos malthusiens et marxistes, Richard Hoffmann rejoint ici Bruce Campbell : les causes biologiques de l'effondrement démographique du milieu du XIV ${ }^{\mathrm{e}}$ siècle étaient imparables et elles n'ont rien à voir avec la « crise du féodalisme » si ce n'est par leur incidence sur la façon dont la société médiévale a construit ses écosystèmes. Pour autant, elle a eu un rôle décisif, limitant les effets de la céréalisation et favorisant des transformations plus rapides et plus profondes qui permettent, avec le retour de l'essor démographique, d'aller vers un nouveau modèle. Une hypothèse intéressante d'Hoffmann est que l'incapacité à comprendre les causes de la « catastrophe » a favorisé dans les esprits les plus ouverts le développement d'un empirisme qui est l'une des sources de la révolution scientifique et de la révolution industrielle, ce qui nous conduit tout naturellement à la « grande divergence ${ }^{8}$ » et au livre de Bruce Campbell dont le propos est de lier celle-ci à la « grande transition».

\section{Priorité au climat}

La démarche de Bruce Campbell est pourtant tout autre et résulte à la fois de plusieurs dizaines d'années de recherche personnelles et de compagnonnages multiples avec des collègues venus d'horizon divers et côtoyés au cours d'une impressionnante suite de programmes interdisciplinaires ${ }^{9}$. Le travail repose en effet sur l'utilisation systématique

7. R. C. Hoffmann, An Environmental History..., p. 237.

8. K. POMERANZ, Une grande divergence : la Chine, l'Europe et la construction de l'économie mondiale, Paris, 2010 [2000].

9. Bruce Campbell les détaille dans une introduction qu'il faut absolument lire (p. xvixxiv). Cette synthèse résulte de l'invitation du Département d'Économie de l'Université de 
de données fournies par les mesures les plus sophistiquées des différentes composantes climatiques et biologiques ${ }^{10}$, y compris celles sur les rendements dans les manoirs anglais établies par Campbell lui-même. Beaucoup sont utilisées pour la première fois dans une telle synthèse. Ces nouvelles sources permettent à l'historien de relire d'un œil neuf et plus précis des événements qui ont déjà fait l'objet de nombreuses analyses. Bruce Campbell peut ainsi dresser un tableau d'une fascinante minutie et d'une grande rigueur de l'écosystème britannique et des implications sociales de son évolution pendant la «grande transition »: la période instable qui s'étend entre d'une part la MCA, the Medieval Climate Anomaly, «l'anomalie climatique médiévale », pendant laquelle la moyenne

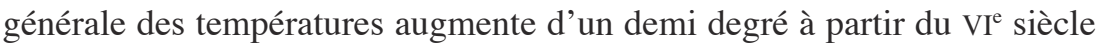
avec un maximum vers $800-900$ puis déclinent doucement jusque vers 1280, et d'autre part le LIA, the Little Ice Age, « le petit âge glaciaire », qui commence vers 1420 et sévit jusque vers 1480 (le minimum de Spörer, avec une baisse d'un demi degré au-dessous de la moyenne). On observe ensuite une brève amélioration, suivie d'un retour dans le LIA qui dure jusque vers 1700. Étant donné la complexité du livre, il m'a paru plus utile de décrire le modèle général présenté ici que d'en donner un compte rendu détaillé, notamment pour ses aspects portant spécifiquement sur l'Angleterre ${ }^{11}$.

Bruce Campbell développe deux analyses parallèles qui se recoupent fréquemment, l'une sur le climat, l'autre sur l'activité microbienne. Pour comprendre l'évolution de l'écosystème européen, il construit une séquence climatique, particulièrement précise pour la période 1320-1420, à partir de courbes et d'histogrammes montrant l'évolution de vingt-quatre indices qui traduisent des données physiques : température moyenne de l'hémisphère nord, largeur des tree-rings (Angleterre, Europe, Asie, Amérique), crues du Nil, spéléothèmes ${ }^{12}$ d'Écosse, humidité de l'air en Asie centrale, précipitations (Écosse, Asie du Sud), etc. Il les confronte ensuite aux courbes des prix et des rendements. L'activité solaire, dont le rayonnement est mesuré par les isotopes cosmogéniques, est la principale cause des variations de température, mais elle n'est pas la seule : les éruptions volcaniques, évaluées à partir des carottes glaciaires, comptent

Cambridge à prononcer les Ellen McArthur Lectures 2013 : «The Great Transition : Climate, Disease and Society in the 13th and 14th Centuries », 4, 6, 11 et 13 février 2013, [en ligne] URL : http://www.econsoc.hist.cam.ac.uk/podcast-campbell.html.

10. Beaucoup de ces données peuvent être consultées sur des sites dédiés comme celui du National Climatic Data Center des États-Unis : URL : www.ncdc.noaa.gov/data-access/ paleoclimatology-data.

11. Voir J.-P. GENET, « À propos de la Grande Transition : mesure et histoire. Bruce M. S. Campbell, The Great Transition... », Histoire \& Mesure, 32/2 (2017 [2018]), p. 165174, [en ligne] URL : https://journals .openedition.org/histoiremesure/6295.

12. C'est-à-dire les concrétions minérales dans les grottes. 
aussi. Le rayonnement, élevé de 800 à 1260, agit sur les océans, et surtout sur le plus vaste, le Pacifique. Les alizés soufflant d'est en ouest chauffent l'eau qui s'évapore et forme les moussons : les eaux profondes, plus froides, remontent à la surface et se réchauffent à leur tour. Mais ce système est instable : l'anticyclone de l'île de Pâques, qui influe sur les alizés, remonte plus ou moins et engendre l'oscillation australe (ENSO, El Niño Southern Oscillation). Elle est positive quand l'eau s'évapore vite à l'ouest du Pacifique, donnant des moussons régulières et abondantes sur l'Inde et l'Asie du Sud-Est alors que la côte ouest de l'Amérique connaît la sécheresse. Par le jeu de la téléconnection, l'oscillation australe influe sur la NAO (North Atlantic Oscillation) : un ENSO positif renforce la NAO, poussant les dépressions océaniques le long des côtes atlantiques, amenant des hivers doux et humides et bloquant l'air glacial de la Sibérie sur l'Est du continent, tandis que la sécheresse frappe les côtes du Maroc et de l'Asie centrale. Cette situation prévaut jusque vers 1280, à l'exception de brèves crises (ainsi, 1202-1204 et 1256-1258 en Angleterre ${ }^{13}$ ), créant ainsi les enabling conditions qui ont favorisé l'écosystème et permis l'essor économique et démographique de l'Europe.

Le rayonnement diminue ensuite, avec deux paliers de baisse vers 1280-1342 et vers 1415-1534 (minimum entre 1430 et 1450, ouvrant le LIA) et, entre ces deux paliers, un bref intervalle de forte irradiation (le maximum « chaucérien » culminant en 1380). De ce fait, l'évaporation des eaux du Pacifique faiblit, et l'ENSO devient négative : l'eau est poussée jusqu'aux côtes d'Afrique et stagne le long des côtes américaines, se déversant sur ces deux zones, tandis que la mousson, peu active en Inde, inonde l'Indonésie. La NAO faiblit à son tour : l'air sibérien descend, provoquant des hivers plus froids et des pluies excessives qui multiplient les mauvaises récoltes dans l'Europe du Nord-Ouest durement frappée par la famine en 1315-1318. En revanche, les côtes marocaines et l'Asie centrale connaissent un climat plus humide. Dans les années 1340-1370, le changement se confirme : les moussons, irrégulières, provoquent des sécheresses en Inde et en Chine, les crues du Nil enflent (maximum en 1341), et la NAO, atténuée, permet aux masses d'air sibériennes de repousser l'anticyclone des Açores au sud. Les

13. B. M.S.CAMPBELL, « Global Climates, the 1257 Mega-eruption of Samalas Volcano Indonesia, and the English Food Crisis of 1258 », Transactions of the Royal Historical Society, 17 (2017), p. 87-121, analyse précisément cette crise, négligée par les historiens obnubilés par les démêlés d'Henri III et des barons. L'évolution des prix, des rendements manoriaux et des tree-rings permet de reconstituer le scénario : un NOS excessif en 1255, 1256 et 1257 provoque des pluies abondantes et continues (selon Matthieu Paris, il plut tous les jours en 1256 du 15 août au 2 février) ; la moisson de 1255 est médiocre, celles de 1256 et 1257 désastreuses. En 1258, conséquences de l'éruption du Samalas (la plus importante connue après celle de Santorin), une soudaine chute des températures et une forte activation du NOS retardent les moissons et aggravent encore la crise et la prolonge. Les fouilles du cimetière de St Mary’s Spital à Londres confirment l'ampleur des ravages de la famine. 
dépressions pluvieuses de l'Atlantique continuent donc à circuler durant le printemps et l'été tandis que la dépression du golfe de Gênes remonte vers le nord, inondant l'Europe centrale, et que dans le Nord les hivers sont très froids et les étés trop humides. L'humidité des steppes asiatiques s'élève encore. Une caractéristique essentielle du climat européen pendant cette période est sa variabilité, mesurée par la variance des températures : la courbe ${ }^{14}$ commence à monter vers 1300 , connaît un pic très élevé entre 1350 et 1390, puis baisse très rapidement à partir de 1395. La réorganisation globale du climat se termine vers 1420 et l'Europe bascule alors dans le LIA avec le minimum de Spörer ; une ENSO négative prédomine, et la faiblesse et l'irrégularité des moussons désorganisent la NAO (au plus bas en 14541455) jusqu'à ce que vers 1480 le rayonnement solaire augmente un peu et améliore la situation.

Les changements climatiques agissent aussi sur les microbes. Dès l'orée du XIV siècle, l'humidité croissante a stimulé des épizooties en Angleterre, où fasciolose et gale du mouton déciment les ovins en 13141316, et en Bohême, où une grande épizootie de peste bovine sévit dès 1314 avant de balayer l'Europe pour atteindre les îles Britanniques en 1319-1320. Mais le phénomène crucial est celui de la peste, disparue en Europe depuis la fin de l'épidémie "justinienne » au $\mathrm{VI}^{\mathrm{e}}$ siècle, qui a bien été provoquée par le bacille de Yersin, une épidémie elle aussi liée à un accident climatique majeur dû à un cataclysme (éruption volcanique, explosion d'une comète ?) survenu en 536. Les conditions générées par la MCA ont ensuite rejeté le bacille dans sa zone d'endémie, en Asie Centrale où les rongeurs (gerbilles de Mongolie, spermophiles et marmottes de la Caspienne) étaient porteurs du bacille de Yersin : les Européens n'avaient donc plus d'immunité contre lui, bien que le commerce international ait intensifié les relations entre Europe et Asie centrale. Or, l'évolution climatique a modifié l'écosystème de l'Asie centrale, les masses d'air devenant de plus en plus humides dès le début du $\mathrm{XIV}^{\mathrm{e}}$ siècle, avec un pic à partir de $1340^{15}$ : l'habitat des rongeurs s'est donc étendu et leur population densifiée. Le bacille de Yersin s'est multiplié, et de l'endémie on est passé à l'épizootie. Les puces des rongeurs, perdant leur support, se sont reportées sur les rats, qui vivent au contact des hommes. Leurs puces (Xenopsylla cheopis) ont été à leur tour contaminées et, les rats mourant de la peste, elles se sont attaquées aux hommes, dont la puce (Pulex irritans) est à son tour devenue le vecteur de la peste bubonique. Ses victimes ont aussi transmis la maladie par voie pulmonaire. Une description méticuleuse fondée sur une bibliographie très technique permet de suivre le passage à l'homme d'un bacille d'autant plus létal qu'il a muté, si l'on se fie aux analyses génomiques, sur le versant chinois de la zone aride, au

14. B. M. S. CAMPBELL, The Great Transition..., p. 203.

15. Ibid., p. 248 : courbe des précipitations en Asie centrale. 
nord-est du plateau Tibétain. Guerre et commerce ont mis Yersinia pestis medievalis sur la route de l'Ouest : une épidémie en Khirgizie (1338) l'annonce, il passe ensuite à Caffa (1346), d'où les vaisseaux génois le transportent en Europe occidentale avec les effets meurtriers que l'on sait. Le refroidissement général des températures et les étés frais et humides qui l'accompagnent au $\mathrm{XV}^{\mathrm{e}}$ siècle offraient au bacille de Yersin des conditions optimales, d'où le retour régulier de l'épidémie et l'effondrement durable de la population jusqu'à un minimum en 1420-1450. La société médiévale a été prise dans the perfect storm ${ }^{16}$ : la pandémie ne doit pas son ampleur à la seule arrivée de Yersinia pestis, mais au fait que cela s'est produit en 1347, quand les conditions climatiques étaient optimales pour sa diffusion.

Comme Richard Hoffmann, Bruce Campbell refuse d'opposer l'endogène (la société) et l'exogène (le monde naturel, climat et biologie), liés par des interrelations que leurs deux livres s'attachent à démêler : il n'entend pas sombrer dans la facilité du déterminisme qui, en faisant du climat le responsable des grandes crises de l'Europe préindustrielle, " échoue à rendre justice à la complexité des actions et des réactions humaines, sans parler de l'autonomie des agents biologiques ${ }^{17}$ ». Mais on peut se demander s'il y parvient. Depuis longtemps, il s'est inscrit en faux contre le «modèle Postan » et la théorie malthusienne en démontrant, aussi bien dans ses recherches sur les rendements ${ }^{18}$ que dans le grand projet auquel il a participé avec Derek Keene sur le ravitaillement de Londres ${ }^{19}$, la capacité d'adaptation et l'aptitude au changement de la société et de l'économie médiévales; et, à juste titre, il observe que le cas anglais n'est pas unique. La société médiévale souffrait du climat et de la peste et de la baisse conséquente du nombre des consommateurs et des producteurs (qui a aussi des effets positifs car elle permet l'abandon des terres à bas rendement et l'augmentation de l'espace dévolu à l'élevage). Mais elle devait aussi compter avec le ralentissement des échanges commerciaux et la fermeture des routes asiatiques, avec le manque de numéraire et de métaux précieux et

16. Ibid., p. 399.

17. Ibid., p. 395 .

18. B. M. S. CAMPBELL, Three Centuries of English Crops Yields, 1211-1491, 2007, [en ligne] URL: http://www.cropyields.ac.uk/, consulté le 15/01/2019.

19. B. M. S. CAmpbell, J. A. Galloway, D. J. KeEne, M. Murphy, A Medieval Capital and Its Grain Supply : Agrarian Production and Its Distribution in the London Region c. 1300, Londres, 1993 (Historical Geography Research Series, 30). 
la baisse des taux d'intérêt (Gregory Clark ${ }^{20}$ et Stephan Epstein ${ }^{21}$ ). Surtout, les ravages des guerres européennes, particulièrement intenses à partir de 1340, aggravent encore la récession par les pertes humaines et matérielles qu'elles entraînent comme par la pression fiscale qu'elles justifient. S'y ajoute la poussée ottomane qui aboutit à la prise de Constantinople en 1453 . Pourtant, grâce inter alia à sa capacité de diversification et d'adaptation pour améliorer rendements et productivité, l'Europe se redresse rapidement à la fin $\mathrm{du} \mathrm{XV}^{\mathrm{e}}$ siècle, et d'abord à partir des pays de la façade atlantique : en Angleterre et plus encore aux Pays-Bas pour les rendements, et surtout en Castille et au Portugal, qui récoltent alors les fruits des explorations maritimes menées depuis le XIV e siècle le long des côtes africaines. Mais tous ces éléments sont juxtaposés, les courbes qui les décrivent superposées, sans qu'il y ait de véritable réflexion théorique pour les faire entrer dans une commune structure, et relier cette structure aux phénomènes naturels si admirablement analysés. De fait, les facteurs socio-politiques sont presque ignorés ${ }^{22}$, comme si la guerre et les mécanismes des prix et de la monnaie n'avaient rien à voir avec le développement des États et les transformations profondes de la culture et de la société politique européenne, tous éléments essentiels à la compréhension de cette période.

\section{Conjoncture ou/et socio-écologie?}

On trouvera certains de ces éléments dans le livre dont John Drendel a dirigé la publication ${ }^{23}$ : il édite les actes d'un colloque tenu à Montréal en 2002, prélude au programme dirigé par Monique Bourin et François Menant sur la conjoncture de 1300 en Méditerranée. Ceux des colloques suivants (Rome 2004 et 2008, Madrid 2005) ont déjà été publiés ${ }^{24}$. Heureusement, plusieurs des articles et les bibliographies ont été remis à jour. L'objectif à Montréal était de débattre des thèses de Maurice Postan et de Georges

20. G. Clark, «The Cost of Capital and Medieval Agricultural Techniques », Explorations in Economic History, 25/3 (1988), p. 265-294; et « The Interest Rate in the Very Long Run : Institutions, Preferences and Modern Growth », 2005, [en ligne] URL : https://www.researchgate.net/publication/228426504_The_interest_rate_in_the_very_long_ run_institutions_preferences_and_modern_growth/download, consulté le 22/01/2019.

21. S. R. EPSTEIN, Freedom and Growth. The Rise of States and Markets in Europe, 1300-1750, Londres/New York, 2000.

22. Voir sur ce point E. CHANEY, « Medieval Origins : A Review Essay on Campbell's The Great Transition », Journal of Economic Literature, 56/2 (2016), p. 643-656.

23. J. Drendel éd., Crisis in the Later Middle Ages. Beyond the Postan-Duby Paradigm, Turnhout, 2015.

24. S. CAROCCI éd., La mobilità sociale nel Medioevo : rappresentazioni, canali, protagonisti, metodi d'indagine, Rome, 2010 ; M. BOURIN, J. DRENDEL et F. MENANT éd., Les Disettes dans la conjoncture de 1300 en Méditerranée occidentale, Rome, 2011 ; M. BoURIN, F. Menant et L. To FIgUERAs éd., Dynamiques du monde rural : échanges, prélèvements et consommation en Méditerranée occidentale, Rome, 2014. 
Duby, et à travers eux des théories malthusiennes et marxistes, celles-ci étant représentées notamment par Robert Brenner et Guy Bois. Si l'on est loin des innovations de Campbell, on retrouve les mêmes hypothèses sur le rôle du climat et de la maladie, pour certaines nées des observations prémonitoires de Robert Fossier, qui avait déjà conclu qu'il fallait dissocier la crise démographique de l'analyse du mode de production, et une remise en cause de la notion de crise, surtout fondée sur l'exemple méditerranéen. La présentation du volume par John Drendel est claire et précise et rend pleine justice à l'article d'Édouard Perroy ${ }^{25}$ qui, tout en soulignant avant Postan l'importance du facteur démographique, laissait entrevoir par son « ciseau » entre prix agricoles et salaires l'importance du rôle des États (guerre et fiscalité) et des marchés. Les trois premiers articles portent sur les marchés et la commercialisation. Ils concluent à la nécessité d'élargir le modèle néo-malthusien de Postan, trop rigide, incapable de générer le changement (contrairement à celui de Duby) et de tenir compte de la diversification induite par la multiplication des marchés ${ }^{26}$, notamment au niveau des petites villes ${ }^{27}$, et négligeant les aspects cycliques de l'investissement ${ }^{28}$. On rattachera à ce groupe l'article de synthèse de John Munro, qui considère le modèle démographique de $\mathrm{M}$. Postan comme inadéquat pour expliquer le mouvement des prix et des salaires, faute d'analyser de façon approfondie les problèmes monétaires ${ }^{29}$.

Dans cette même perspective de discussion du modèle Postan, trois articles traitent du monde paysan. Philip Schofield souligne que c'est la « tyrannie de son propre modèle » qui a interdit à Postan de l'élargir pour y inclure les aspects aujourd'hui mis en avant pour le combattre, le crédit, le marché et la commercialisation, sujets dont il était pourtant un éminent spécialiste $^{30}$. Anne DeWindt, à partir du cas anglais, propose le concept d'agency (qu'on pourrait traduire par « capacité d'agir ») pour montrer comment les paysans étaient capables d'initiative et d'innovation pour surmonter les obstacles ${ }^{31}$. Enfin, Erik Thoen et Tim Soens montrent que

25. É. Perroy, « À l'origine d'une économie contractée : aux origines des crises du XIVe siècle », Annales E.S.C., 4/2 (1949), p. 167-182.

26. R. BRITNELL, "Commercialization, Stagnation and Crisis, 1250-1350 », dans J. DRENDEL éd., Crisis in the Later Middle Ages..., p. 15-34.

27. C. DYER, «Medieval Small Towns and the Late Medieval Crisis », dans J. DRENDEL éd., Crisis in the Later Middle Ages..., p. 35-52.

28. J. LANGDON, « The Long Thirteenth Century : an Era of Schumpeterian Growth », dans J. DRENDEL éd., Crisis in the Later Middle Ages..., p. 53-71.

29. J. MunRo, « Money Matters. A Critique of the Postan Thesis on Medieval Population, Prices, and Wages », dans J. DRENDEL éd., Crisis in the Later Middle Ages..., p. 127-194.

30. P. SCHOFIELD, «M. M. Postan and the Peasant Economy », dans J. DRENDEL éd., Crisis in the Later Middle Ages..., p. 73-93.

31. A. DeWInDT, « Historians and Peasant Agency : Studies of Late Medieval English Peasants », dans J. Drendel éd., Crisis in the Later Middle Ages..., p. 95-125. 
même en Flandre la commercialisation n'explique pas tout et que deux modèles divergents se sont développés, en fonction de l'évolution des rapports de domination entre les paysans et les classes dirigeantes, d'ailleurs plus ou moins concurrentes (le comte, les bourgeois, les différents groupes de l'aristocratie féodale) : la CSE (Commercial Survival Economy) en Flandre intérieure, caractérisée par la prédominance de la petite exploitation adaptée à la survie des familles, et la CBE (Commercial Business Economy) en Flandre côtière, où la propriété paysanne a décliné au profit de fermes dépendant de grands propriétaires seuls susceptibles de supporter les investissements exigés pour maintenir les infrastructures face aux désastres écologiques ${ }^{32}$.

Le dernier tiers du volume est constitué par six études de cas qui se rattachent plus ou moins directement au débat. Thierry Pécout donne une brève analyse historiographique de la crise en Provence, dans le sillage des travaux de Georges Duby ${ }^{33}$. Monique Bourin s'attache à montrer, en écho aux travaux anglais, les « nouveaux chemins de développement » du Languedoc d'avant la peste : surgissement des vignes, essor d'un véritable marché foncier où les artisans sont de plus en plus présents, développement du crédit et de l'industrie textile (draperie notamment). Si crise il y a, c'est au sens de transformation rapide, la prospérité étant loin d'avoir disparu ; le Roussillon et le royaume de Valence offrent une image similaire ${ }^{34}$. Pour sa part, Philippe Bernardi explore ce qui n'est tout au plus que «litote » dans le modèle postanien, les revenus non agricoles, à partir de l'exemple du marché des matériaux de construction (bois, pierre, chaux, plâtre) produits dans les campagnes - ce qui dispense d'une opposition stérile entre artisanat et agriculture. Les prix de ces matériaux et de leur transport, bien connus grâce aux comptes d'Avignon, connaissent une formidable augmentation juste après la peste et l'auteur suggère que l'activité artisanale ou même industrielle a occupé une place de plus en plus importante dans les campagnes ${ }^{35}$. Francine Michaud interroge les sources notariales pour décrire la condition des travailleurs à Marseille avant et après la Peste : si celle-ci a fait décliner la proportion des prolétaires pauvres, l'embellie n'a

32. E. Thoen et T. Soens, « The Family or the Farm : A Sophie's Choice ? The Late Medieval Crisis in Flanders », dans J. Drendel éd., Crisis in the Later Middle Ages..., p. 195-224.

33. T. PÉCOUT, « Le destin heuristique d'une histoire de la panique. La crise en Provence au Moyen Âge finissant : jalons historiographiques pour le XIV e siècle », dans J. DRENDEL éd., Crisis in the Later Middle Ages..., p. 235-249.

34. M. BouRIN, « De nouveaux chemins de développement dans le Languedoc d'avant la peste », dans J. DRENDEL éd., Crisis in the Later Middle Ages..., p. 251-272.

35. P. BERNARDI, «Quand le bâtiment va... Une facette des rapports ville-campagne : le marché des matériaux », dans J. DRENDEL éd., Crisis in the Later Middle Ages..., p. 273-296. 
été que de courte durée ${ }^{36}$. Laure Verdon analyse les capbreus (terriers), une source exceptionnelle pour la population et les structures de la propriété dans les villages du Roussillon, et montre comment seigneurs et tenanciers ont développé des stratégies innovantes (fractionnement des manses, accapte, sous-location, association pro indiviso) pour s'adapter à une pression démographique croissante ${ }^{37}$. Enfin, Constance Berman présente le cas des nonnes cisterciennes de la région parisienne, dont l'insolente prospérité est à elle seule un défi aux thèses de Postan : malheureusement pour elles, elle n'échappa pas à la branche masculine de l'ordre qui siphonna sans vergogne leurs richesses pour parer à ses propres difficultés ${ }^{38}$.

Mais quelle que soit l'ampleur de cette moisson, les ouvrages de Richard Hoffmann et de Bruce Campbell montrent qu'un nouveau paradigme s'impose désormais à l'histoire économique, la perspective écologique au sens le plus large du terme, qui offrent désormais à l'historien un cadre chronologique relativement fiable et des séries de mesures qui permettent de construire des démonstrations plus rigoureuses. Le livre d'Hoffmann vaut surtout comme introduction à cette nouvelle perspective, mais celui de Bruce Campbell s'impose déjà comme un livre fondamental, compagnon indispensable à tout travail de recherche sur l'économie et la société médiévales. En fait, et c'est pourquoi le choix du terme «perspective » n'est pas indifférent, l'un de ses mérites est d'ouvrir de nouvelles pistes à la recherche. Mais Bruce Campbell écrit :

L'économie classique, la théorie marxiste et la nouvelle économie institutionnelle ont tous permis d'approfondir la compréhension de la Grande Transition [...] Aucune [de ces approches] n'a cependant réussi à donner un récit historiquement convaincant de cette transformation socio-écologique, chronologiquement étendue et géographiquement extensive ${ }^{39}$.

Ce récit, dans le cadre qu'il s'assigne, le cadre socio-écologique, Bruce Campbell l'a magistralement donné : mais, de son propre aveu, l'écologie ne suffit pas à « faire » la société, bien d'autres éléments interviennent, qui ressortent de la culture au sens large, du politique, ou des rapports de domination au sein de la société, et sur ces points, il serait peutêtre bon de revenir vers la réflexion théorique, notamment en s'inspirant des travaux des anthropologues et des sociologues, surtout si l'on veut donner

36. F. MiCHAUD, «Réflexions sur la condition des travailleurs au cours du XIV ${ }^{\mathrm{e}}$ siècle à Marseille », dans J. DRENDEL éd., Crisis in the Later Middle Ages..., p. 297-322.

37. L. VERDON, « Les capbreus royaux roussillonnais et la crise malthusienne », dans J. DRENDEL éd., Crisis in the Later Middle Ages..., p. 323-338.

38. C. H. BERMAN, « Cistercian Agriculture in Female Houses of Northern France, 1200$1300 »$, dans J. Drendel éd., Crisis in the Later Middle Ages..., p. 338-363.

39. B. M. S. CAMPBELL, The Great Transition..., p. 395. 
à l'Europe médiévale la place qu'elle doit occuper dans l'historiographie en construction de la «Grande Divergence », comme le souhaite d'ailleurs Bruce Campbell lui-même. Au moins a-t-il levé une lourde hypothèque : les éventuels blocages économiques et démographiques de la société médiévale n'ont pratiquement rien à voir avec le déclenchement de la Peste noire et l'effondrement de la population européenne qui ont suivi. Ainsi redéfini, le débat sur la crise (où la transformation ?) de la société médiévale de la fin du Moyen Âge peut et doit désormais reprendre sur des bases nouvelles.

Jean-Philippe Genet - Université Paris 1 Panthéon-Sorbonne

De la " grande crise " à la " grande transition " : une nouvelle perspective ? Crise, climat, histoire environnementale, maladie, peste.

From the "great crisis" to the "great transition" : A new perspective?

Crisis, climate, disease, environmental history, plague. 